Itinéraires Itinéraires

Littérature, textes, cultures

2010-2 | 2010

Les blogs

\title{
Le blog intime au croisement des genres de l'écriture de soi
}

\section{Oriane Deseilligny}

\section{(2) OpenEdition}

\section{Journals}

Édition électronique

URL : http://journals.openedition.org/itineraires/1985

DOI : 10.4000/itineraires.1985

ISSN : 2427-920X

Éditeur

Pléiade

\section{Édition imprimée}

Date de publication : 1 juillet 2010

Pagination : 73-82

ISBN : 978-2-296-12012-9

ISSN : 2100-1340

\section{Référence électronique}

Oriane Deseilligny, «Le blog intime au croisement des genres de l'écriture de soi », Itinéraires [En ligne], 2010-2 | 2010, mis en ligne le 01 juillet 2010, consulté le 03 mai 2019. URL : http://

journals.openedition.org/itineraires/1985; DOI : 10.4000/itineraires.1985

\section{(ब) $(\Theta \Theta$}

Itinéraires est mis à disposition selon les termes de la licence Creative Commons Attribution - Pas d'Utilisation Commerciale - Pas de Modification 4.0 International. 


\title{
Le blog intime au croisement des genres de l'écriture de soi
}

\begin{abstract}
If we consider the use of the Internet and the practice of writing, weblogs have followed personal websites. In this paper, we examine the way by wich those electronic writing practices have inherited some discursive and morphological forms from classical genres, namely diaries and correspondence. Diary and intimate correspondence are two genres of self-writing, the evolutions of which are linked. Thus, weblogs are in line with the concept of self-writing defined by Michel Foucault.
\end{abstract}

Keywords : diary, letter, writing the self, genre, weblog

Mots clés : journal personnel, lettre, écriture de soi, genre, blog

Massivement utilisés à partir de 2002-2003 comme dispositifs d'autopublication, les blogs sont intervenus dans un second temps de l'histoire d'Internet et de son appropriation par les usagers. Avant eux les sites personnels - aux pages élaborées de toutes pièces par les internautes - ont été des espaces de construction de l'identité narrative ${ }^{1}$ et de mise en forme de soi. Si nombre de discours médiatiques ont pu voir dans les blogs une « révolution » radicale des modes d'écriture et de participation au débat public, nous nous situons ici dans une approche inverse. Car l'engouement massif à l'endroit des blogs - et plus profondément ce qui a pu être analysé comme relevant d'un « individualisme expressif contemporain ${ }^{2} \gg-$ n'a pas surgi ex nihilo, à la faveur d'innovations techniques. Certes, comme dispositif éditorial et communicationnel, le blog est bien sans précédent dans l'histoire des supports. Toutefois sur le plan discursif, il hérite de formes ancrées dans l'histoire longue de la

1. Annabelle Klein, «Les homepages, nouvelles écritures de soi, nouvelles lectures de 1'autre », Spirale, n 28, 2001, p. 67-82.

2. Laurence Allard et Frédéric Vandenberghe, «Express yourself! Les pages perso entre légitimation technopolitique de l'individualisme expressif et authenticité réflexive peer to peer », Réseaux, $\mathrm{n}^{\circ} 117,2003$, p. 191-219. 
culture écrite. Nous nous proposons de montrer dans quelle mesure, comme format spécifique de publication et comme structure de production textuelle préformatée, le blog réinvestit et automatise des formes textuelles, discursives et de communication écrite bien plus anciennes liées à l'écriture de soi ${ }^{3}$.

Avant l'apparition des blogs, dans un ensemble très diversifié et hétéroclite de pages personnelles, les journaux intimes publiés en ligne ont en effet constitué une première étape décisive dans le développement d'une écriture de soi sur le $\mathrm{Web}^{4}$ et dans l'expérimentation de formes de subjectivation utilisant l'outil numérique. Nombre de ces sites s'inscrivaient dans le prolongement d'un journal manuscrit puisque parmi les pionniers du journal en ligne figuraient des diaristes attachés au papier qui ont transféré leur pratique sur le médium numérique. Rejoints ensuite par des individus séduits par cette forme nouvelle d'écriture de soi médiatisée et rassemblés en communautés de diaristes numériques ${ }^{5}$, ils ont pendant quelques années été moins de trois cents sur le Web francophone.

À partir de 2003, la simplification des outils de publication en ligne, l'apparition des systèmes de gestion de contenu - et au premier chef des blogs - a permis aux internautes néophytes de publier des contenus sans avoir besoin de coder eux-mêmes leurs pages en langage HTML. L'exaltation considérable pour ces dispositifs de publication est sans conteste liée à leur facilité d'utilisation et à l'interactivité permise avec le lecteur. Cette dernière a en effet transformé le modèle communicationnel puisque dans les sites personnels de la première heure, les lecteurs ne pouvaient pas agir sur le site qui demeurait assez statique - au contraire des blogs dans lesquels ils peuvent désormais rédiger des commentaires sous les billets.

Afin de mieux saisir les pratiques actuelles et les formes textuelles que le blog, comme forme éditoriale, mobilise, nous étudierons la manière dont les premiers journaux personnels publiés en ligne ont eux-mêmes auparavant cristallisé des formes d'écriture liées au diarisme mais également à une autre catégorie de discours relevant de l'écriture de soi, le genre épistolaire. De fait, les pratiques d'écriture qui ont marqué l'appropriation du Web par le grand public se sont situées à la fois dans un processus mimétique par rapport aux usages manuscrits du journal et innovant par les cadres logiciels mobilisés.

Aussi examinerons-nous la textualité des journaux personnels publiés sur le Web avant 2003, en tant qu'elle est révélatrice d'un rapport spécifique à l'écriture de soi dont les blogs héritent. Hétérogène et composite, cette

3. Michel Foucault, «L'écriture de soi », Corps écrit, n ${ }^{5}$, février 1983, p. 3-23; repris dans Dits et écrits (1976-1988), t. II, Gallimard, coll. « Quarto », 2001, p. 1234-1249.

4. Oriane Deseilligny, L'écriture de soi, continuités et mutations. Du cahier aux journaux personnels sur le Web (1998-2003), thèse de doctorat en Sciences de l'information et de la communication, université Paris X-Nanterre, 2006.

5. Philippe Lejeune, « Cher écran... ». Journal personnel, ordinateur, Internet, Paris, Seuil, coll. « La couleur de la vie », 2000. 
textualité numérique met en question la notion de genre et son efficacité pragmatique dans l'identification des formes et des discours. On abordera ici le genre en faisant l'hypothèse que c'est l'interaction permanente entre des dispositifs d'écriture, des supports d'information et des situations de communication qui fait travailler les genres comme référents discursifs et rhétoriques. Dans une perspective communicationnelle, qui souscrit en outre aux propositions de Jean-Marie Schaeffer ${ }^{6}$, la dimension énonciative, textuelle et pragmatique tout autant que le contexte communicationnel participent selon nous à la démarche de catégorisation.

Dans une approche généalogique et littéraire, nous commencerons donc par identifier précisément les formes énonciatives et morphologiques référées à des genres de discours antérieurs que le journal personnel en ligne donne à appréhender à l'écran. Dans un second temps, nous inscrirons cet héritage dans une histoire plus longue encore, celle des trajectoires croisées de deux genres relevant plus globalement du champ de l'écriture de soi : la lettre familière et le journal intime. Cette filiation historique nous permettra enfin de spécifier les formes énonciatives et discursives instanciées dans le dispositif blog et d'en interroger l'imaginaire.

\section{Le journal en ligne et la rhétorique épistolaire}

\section{L'image du texte : entre lettre et journal?}

La spécificité de la textualité composant les journaux personnels en ligne se donne à appréhender, en première approche, à travers l'étalement du texte dans l'espace graphique. À travers la notion d'énonciation éditoriale $^{7}$, Emmanuël Souchier a développé l'idée d'une co-détermination entre l'image du texte et son contenu discursif. Certaines catégories de discours sont en effet identifiables, entre autres, par les spécificités de leur mise en texte ou de la mise en page. D'emblée pourtant, la morphologie globale du journal personnel en ligne est marquée par une certaine confusion dans l'agencement des formes et des catégories discursives à l'écran. La part visuelle du texte semble renvoyer en effet autant à la tradition manuscrite et ancienne du journal qu'au protocole graphique de la lettre familière. Tout se passe comme si chaque mise à jour donnait à voir à la fois un journal et une lettre.

Ces journaux personnels en ligne sont généralement construits sur une structure divisée en quatre parties principales : i) la datation de chaque entrée, éventuellement accompagnée d'un titre, ii) un en-tête initial, iii) un développement, iv) une chute ou un envoi. Hormis le fait de donner un

6. Jean-Marie Schaeffer, Qu'est-ce qu'un genre littéraire?, Paris, Seuil, 1989.

7. Emmanuël Souchier, "L'image du texte, pour une théorie de l'énonciation éditoriale », Les Cahiers de médiologie, ${ }^{\circ}$ 6, 1998, p. 137-145. 
titre à une entrée, on retrouve là des spécificités morphologiques classiques du journal, dont les entrées sont effectivement maillées sur le calendrier et parfois accompagnées d'un en-tête. Au sein de l'espace graphique, les blocs de texte se détachent visuellement dans une logique de différenciation des formes énonciatives. Or, cette distinction graphique et morphologique des énoncés caractérise aussi la rhétorique épistolaire articulée autour de : i) la datation, ii) les séquences d'ouverture et de fermeture, iii) l'identification de l'énonciateur en bas de la page.

Nombre d'entrées des journaux personnels sont disposées sur la pageécran selon une structure qui réfère certes à la morphologie classique du journal, mais qui n'est pas sans évoquer aussi celle de la lettre. Les deux genres ont en commun de déployer une énonciation à la première personne du singulier, datée dans le temps et de surcroît fragmentée. En revanche, sur le plan du discours, les deux genres sont d'ordinaire opposés, puisque le journal est a priori dominé par un régime autarcique (un discours de soi à soi, réflexif) alors que la lettre est transitive, portée par un geste d'adresse à un destinataire autre que soi.

On touche là au cœur de la complexité du texte du journal en ligne dans la mesure où l'image du texte interroge la nature du geste qui fonde la publication en ligne d'un écrit habituellement considéré comme personnel. Cette apparente hybridation des formes qui se manifeste dans les journaux personnels en ligne mais aussi dans les blogs semble indiquer la possibilité d'un destinataire extérieur dans un processus d'écriture de soi. Au-delà de l'agencement visuel des formes, examinons à présent les éléments discursifs qui inscrivent le journal en ligne dans un régime interactionnel assumé.

\section{Formes discursives empruntées à la communication épistolaire}

En premier lieu, on remarque des formules d'en-tête et d'envoi qui adressent explicitement le journal à un destinataire extérieur. Par exemple, les séquences d'ouverture de la mise à jour consistent en des formules relevant du paradigme épistolaire : « coucou!! », « bonjour tout le monde », « cher réseau ». De même, les séquences de fermeture mobilisent des formules d'envoi fréquentes dans la communication écrite : «A + », «A bientôt », « je vous laisse », « bisous », etc.

Ensuite, au-dessous de certaines entrées, on trouve parfois des énoncés assimilés à des post-scriptum, qui corroborent l'idée que le journal en ligne va bien au-delà d'un processus clos de dialogue de soi à soi. Ainsi de cette diariste, qui écrit, en ajout à sa mise à jour « P.-S. Un peu beaucoup par paresse, j'ai décidé de laisser mes entrées du mois sur une même page. Si cela vous gêne, n'hésitez pas à me le dire ». En marge du récit de soi du corps de l'entrée, les diaristes s'autorisent une parole ostensiblement adressée à leurs lecteurs. 
Sur le plan strictement discursif enfin, les reprises diaphoniques présentes dans les journaux en ligne inscrivent également le texte dans une dynamique interactionnelle. Dans le cadre d'un dialogue ou d'une communication écrite, ces dernières désignent diverses formes de reformulation ou d'emprunts faits à partir d'un discours ${ }^{8}$. Si de telles reformulations étaient déjà repérables dans les journaux personnels en ligne, elles le sont encore plus dans les blogs puisque les lecteurs réagissent, dans les commentaires, aux propos tenus par les blogueurs dans leurs billets. Par exemple dans le blog de Lou, un internaute écrit : « Je me disais bien qu'il y avait tempête sous un crâne pour que tu restes ainsi silencieuse. » Commentant le commentaire, la diariste répond à ce lecteur en reprenant son expression : « Oui, mais une toute petite tempête quand même. » Dans l'espace des commentaires, le dialogue prend la place du monologue dialogique qui caractérise les billets du blog.

En « empruntant » à l'épistolaire des formes de discours spécifiques, le journal en ligne manifeste visuellement le travail d'une écriture de soi qui, sur le médium numérique, intègre et assume des destinataires extérieurs. À la communication de soi à soi ordinairement recherchée dans le journal papier s'ajoute - et non pas se substitue - une communication à l'autre dans le journal en ligne. Mais ce qui se donne en surface comme un mélange des genres est à mettre en relation avec des perméabilités beaucoup plus profondes entre la pratique de la lettre et celle du journal intime. Pour expliquer en effet cette prégnance de formes interactionnelles directes dans les journaux personnels en ligne et poursuivie ensuite dans les blogs, il convient à présent de revenir sur l'histoire culturelle et littéraire de ces deux genres de discours.

\section{Journal et lettre, deux formes de l'écriture de soi}

\section{Retour sur le concept d'écriture de soi}

Le rapprochement que nous effectuons entre le journal intime et la lettre comme procédant d'une dynamique commune s'inscrit dans le cadre théorique de l'écriture de soi, telle qu'elle a été développée par Michel Foucault à propos des techniques de subjectivation antiques. Au-delà du contexte dans lequel il examine précisément ces «techniques de soi », Foucault observe la manière dont le Sujet se construit au cours des siècles - notamment à travers un ensemble de pratiques réelles, " analysables historiquement », dont l'écriture est partie prenante.

Dans la culture antique, le souci de soi - ou epimeleia heautou - est un principe fort lié au gouvernement de soi qui procède d'un art de vivre

8. Eddy Roulet définit la diaphonie comme ce qui consiste à « reprendre et à réinterpréter dans son propre discours la parole du destinataire, pour mieux enchaîner sur celle-ci ». Voir Eddy Roulet et al., L'Articulation du discours en français contemporain, Berne, Peter Lang, coll. «Sciences pour la communication», 1985, p. 70-71. 
(technê tou biou). Il organise aussi bien un ensemble d'activités concrètes autour de l'écriture et de la lecture qu'une pratique de l'âme fondée sur des techniques de mémorisation, de méditation, d'examens de conscience, de silence. Pour autant, loin d'éloigner le sujet du monde et des autres, il est une " véritable pratique sociale », un « intensificateur des relations sociales ${ }^{9}$ ». Ce souci de soi, qui exige une conversion du regard que le sujet porte sur lui-même, constitue pour Foucault un « phénomène culturel d'ensemble ${ }^{10}$ » dans toute l'Antiquité mais qui, au-delà, nourrit la philosophie occidentale et la conception du Sujet.

Dans ce contexte, l'écriture apparaît comme une étape essentielle d'un processus d'entraînement de soi par soi. Elle joue un rôle d'épreuve de vérité et prend deux formes principales : les hypomnêmata et la correspondance. Bien que différentes dans leur forme et dans leur destinataire, ces deux pratiques relèvent de la subjectivation. À travers les carnets (hypomnêmata) sur lesquels l'individu note quotidiennement des fragments de choses lues ou entendues, de discours prononcés ou retenus, il s'agit d'équiper le sujet afin qu'il établisse un rapport à soi « aussi adéquat et parfait que possible ». La correspondance est aussi l'espace d'un entraînement de soi par soi, mais cette fois-ci sous le regard du destinataire. À ce titre, la lettre implique une introspection non pas tant comme « déchiffrement de soi par soi que comme une ouverture qu'on donne à l'autre sur soi-même ${ }^{11}$ ». Pour Foucault, les premières formes de récit de soi se trouvent donc moins dans les hypomnêmata que dans les lettres échangées avec autrui.

Bien que nous ne puissions pas développer ici toute la fécondité de l'approche foucaldienne du souci de soi et des techniques de subjectivation, retenons ce rapprochement a priori improbable entre deux formes d'écriture distinctes. Mais le paradoxe ne résiste pas longtemps dès lors que l'on considère davantage le geste spéculaire et mémoriel que l'adresse officielle du texte. Car dans la lettre comme dans un journal, la réflexivité peut l'emporter sur la destination et vice-versa. En d'autres termes, là où la lettre échangée avec un ami peut remplir la fonction d'un journal, ce dernier peut lui-même être adressé à d'autres que soi. Par conséquent, nous sortons d'une perspective générique pour considérer le geste de subjectivation en amont du texte produit et du dispositif formel qu'il instaure.

Rapportée d'abord aux rapports entre lettre familière et journal intime, cette perspective de l'écriture de soi envisage donc moins la destination et les modalités énonciatives que le travail du sujet dans l'écriture, donc dans la connaissance de lui-même référée à la constitution d'une mémoire.

9. Frédéric Gros, «Situation du cours », dans Michel Foucault, L'Herméneutique du sujet. Cours au Collège de France. 1981-1982, Paris, Gallimard/Seuil, coll. "Hautes études », p. 517.

10. Michel Foucault, ibid., p. 11.

11. Michel Foucault, L'Écriture de soi, op. cit., p. 1242. 
Ensuite, à l'épreuve des journaux personnels en ligne et des blogs, parler d'écriture de soi permet de saisir, par-delà la nouveauté technologique et les genres de discours, des indices de permanence et de transformation dans les formes de subjectivation.

\section{Trajectoires croisées de la lettre et du journal}

Sur le Web, ce que les diaristes eux-mêmes définissent comme un « journal en ligne », se caractérise, rappelons-le, par un apparent entremêlement formel. L'écran exhibe là un système complexe de formes que nous allons maintenant tenter d'éclairer historiquement, car les pratiques d'écriture actuelles tendent à exalter une interpénétration séculaire entre deux genres de l'écriture de soi que sont la lettre et le journal.

Outre les liens forts décrits par Michel Foucault entre la lettre et l'écriture pour soi (mais non pas « intime ») que représentaient les hypomnêmata, il faut évoquer le XVIII ${ }^{e}$ siècle, au cours duquel les itinéraires de la lettre familière et du journal intime se croisent. Dans sa forme moderne et laïque, le journal intime est un genre jeune. Beaucoup plus ancienne, de son côté, la lettre familière tend à prendre ses distances au XVIII ${ }^{\mathrm{e}}$ siècle avec les exigences du «naturel feint » qui avaient marqué l'âge d'or de la conversation ${ }^{12}$ et la rhétorique épistolaire sévignéenne. Les études sur l'épistolaire font état d'un net infléchissement de l'esthétique de la " négligence recherchée » dans les lettres, au profit de la transcription progressive des états intérieurs du moi. Lentement, dans cette période qui voit l'émergence de la subjectivité désormais fondée sur l'expression du je par lui-même, la lettre se met au service de l'expression des confidences, des sentiments personnels ${ }^{13}$. Rappelant que « c'est pendant la seconde moitié du XVIII' siècle que l'idée est venue de s'adresser à soi-même par écrit les confidences qu'on réservait jusqu'alors à un ami intime ${ }^{14} »$, Philippe Lejeune décrit le journal comme une « intériorisation de la lettre familière ». De même, Françoise Simonet-Tenant évoque le journal du début du XIX ${ }^{\mathrm{e}}$ siècle comme «l'asymptote de la lettre familière ${ }^{15}$ ».

On observe ainsi un chassé-croisé entre les deux genres, mais aussi une complémentarité et des décalages entre le protocole discursif mis en place et la destination réelle du texte. Au XVIII ${ }^{\mathrm{e}}$ siècle, le glissement de la lettre au

12. Emmanuel Godo, Une histoire de la conversation, Paris, PUF, coll. «Perspectives littéraires », 2003.

13. Marie-Claire Grassi, L'Art de la lettre au temps de La Nouvelle Héloïse et du romantisme, Genève, Slatkine, 1994.

14. Philippe Lejeune et Catherine Bogaert, Un journal à soi. Histoire d'une pratique, Paris, Seuil, coll. « Textuel », 2003.

15. Françoise Simonet-Tenant, « Lettre et journal : ressemblances et hybridations », communication prononcée lors du séminaire de l'AIRE, «Lettre et journal », université Paris 7, octobre 2005. Voir aussi Françoise Simonet-Tenant, «À la recherche des prémices d'une culture de l'intime », Itinéraires. Littérature, textes, cultures, 2009, nº4, p. 39-62. 
journal, avéré dans les écrits littéraires comme dans les pratiques ordinaires se manifeste donc par l'intégration, au sein de la structure dialogique propre à la lettre, de formes réflexives au travers desquelles le scripteur finit ainsi parfois par ne plus s'adresser qu'à lui-même. Plus profondément, c'est la dimension intrinsèquement composite du journal comme de la lettre qui est soulignée. Si le journal peut être défini comme « circulatoire » par le mélange des formes discursives qu'il révèle (conversation, monologue, correspondance, récit), la lettre apparaît aussi comme une écriture « ambulatoire », « qui migre constamment d'une forme à l'autre et ignore les frontières génériques ». Pour Philippe Lejeune, « il n'y a pas une essence éternelle de la lettre, mais l'existence fluctuante et contingente d'un certain mode de communication par écrit, qui, combiné avec d'autres traits, a pu remplir des fonctions différentes dans des systèmes différents ${ }^{16} \gg$.

Dans le champ de l'écriture de soi, une certaine porosité marque historiquement les genres de discours. Ce débordement des catégories génériques renforce l'idée foucaldienne qu'une tension entre communication et réflexivité est constitutive des écrits personnels. L'appréhension de ce champ dans une perspective moins générique que centrée sur la construction de la subjectivité nous mène ainsi à voir dans l'apparente confusion formelle que l'écran exhibe à travers les journaux personnels en ligne, une caractéristique fondamentale de l'écriture personnelle. Par là, nous subsumons les genres de discours historiques de la lettre et du journal sous l'écriture de soi comme modalité d'expression du sujet, inscrite dans des protocoles communicationnels diversifiés.

\section{Le blog, une structure éditoriale aux formes héritées}

À travers le cas des journaux personnels en ligne, apparaît très nettement la disjonction qui peut exister entre le cadre communicationnel et pragmatique dont se dote l'énonciateur et la réalisation effective du message au plan sémantique et syntaxique ${ }^{17}$. L' « iconicité » du journal en ligne fait valoir un double rattachement générique qui procède d'un geste médiatisé d'écriture de soi. Entérinant des chassés-croisés séculaires entre les genres de l'écriture de soi, les blogs héritent de formes qui sont également métamorphosées par l'outil numérique.

En effet, en 2002-2003, les blogs se caractérisent par une forme éditoriale et logicielle rigide, fondée sur une nette séparation des champs communicationnels (billets du blogueur vs commentaires des lecteurs) et l'ancrage très précis de l'énonciation (chaque billet étant accompagné de l'heure du billet et du nom du blogueur). L'architecture commune à toutes les plates-formes d'autopublication est alors marquée par l'automatisation logicielle des coordonnées énonciatives

16. Philippe Lejeune, Le Pacte autobiographique [1975], Paris, Seuil, 1996, p. 315-316.

17. Jean-Marie Schaeffer, op. cit. 
et temporelles que nous avons identifiées comme relevant du geste d'écriture de soi, par-delà les différences génériques. À côté de l'interactivité et des blogrolls qui spécifient ces formats logiciels comme proprement numériques, la datation des billets ainsi que la mention systématique du pseudonyme du blogueur constituent des identifiants forts du dispositif « blog » dont les sources plongent dans les formes anciennes de l'écriture de soi.

Sur cette base, la forme blog constitue un artefact logiciel et logistique empruntant à des techniques anciennes de subjectivation des traits identifiés par les éditeurs de blogs comme suffisamment pérennes et personnalisables pour des appropriations diversifiées en termes de genres de discours et de formes médiatiques (blogs journalistiques, citoyens, cuisine, etc.). Au-delà de ces marqueurs temporels et énonciatifs, le blog se prête désormais à des appropriations et des genres de discours plurimédiatiques qui dépassent largement le contexte de l'écriture de soi. Cependant, en faisant de tout type de discours un texte systématiquement maillé sur le calendrier et référé à un énonciateur unique, ils promeuvent un « moule à texte ${ }^{18}$ » qui se donne comme évident, naturel, mais qui n'en demeure pas moins régenté par des cadres instituants qui ne sont pas neutres.

À travers cette mise en perspective des genres de l'écriture de soi et de certaines formes textuelles composant l'énonciation dans les blogs, nous avons tenté de montrer que ces identifiants majeurs sont au départ issus d'une longue tradition du geste spéculaire, incarnée à travers le journal et la lettre. Comme d'autres formes avant eux, le journal personnel en ligne puis les blogs se situent à l'articulation de la réflexivité et de la transitivité. Ils cristallisent ainsi des dynamiques et des jeux de tensions qui nourrissent depuis longtemps l'écriture de soi, toujours tendue entre le soi et l'autre.

Les évolutions techniques et les appropriations par les internautes ont depuis 2003 largement évolué et contribuent à métamorphoser la forme blog initiale. Pourtant le blog demeure connoté par le genre intimiste : certains médias continuent à le définir comme un " journal extime », un « carnet web » alors même que les contenus multimédiatiques dont il est investi désormais rompent parfois radicalement avec une approche herméneutique du sujet, ou une construction de l'identité du sujet par l'écriture. Au demeurant, la persistance de ces connotations dans l'imaginaire du Web renforce l'idée d'un continuum dans les formes d'écriture de soi par-delà les époques et les genres de discours mobilisés. Ainsi que l'énonçait Michel Foucault, « il y a une technologie de la constitution de soi qui traverse les systèmes symboliques tout en les utilisant ${ }^{19} »$. Dans ses formes intimes

18. Valérie Jeanne-Perrier, «Des outils d'écriture aux pouvoirs exorbitants? », Réseaux, $\mathrm{n}^{\circ} 137,2006$, p. $97-131$.

19. Michel Foucault, «À propos de la généalogie de l'éthique : un aperçu du travail en cours », Dits et Écrits II, Paris, Gallimard, coll. « Quarto », 2001, p. 1447. 
et dans une certaine continuité par rapport à d'autres dispositifs, le blog constitue selon nous un avatar moderne de cette technologie de soi, dans lequel se rencontrent désir de réflexivité et besoin de transitivité au service de la construction de la subjectivité.

$$
\begin{array}{r}
\text { Oriane Deseilligny } \\
\text { Université Paris } 13-\text { CELSA - GRIPIC }
\end{array}
$$

